\title{
Incidence and characteristics of heart block after heart surgery in pediatric patients: A multicenter study
}

\author{
Leonardo Liberman, MD, ${ }^{\mathrm{a}}$ Eric S. Silver, MD, ${ }^{\mathrm{a}}$ Paul J. Chai, MD, ${ }^{\mathrm{b}}$ and Brett R. Anderson, MD, MBA ${ }^{\mathrm{a}}$
}

\section{ABSTRACT}

Background: Advanced second- or third-degree heart block has been reported with variable incidence after surgery for congenital heart disease in children. We report the incidence of heart block requiring a pacemaker and describe the risk factors for this complication in a large multicenter study.

Methods: We performed a retrospective cohort study, using the Pediatric Health Information System database from 45 hospitals in the United States, for all children aged 18 years, discharged between January 1, 2004, and December 31, 2013, who underwent open surgery for congenital heart disease. Patients who had heart block and placement of a pacemaker during the same hospitalization were identified. Demographic characteristics, procedure and diagnostic codes, length of stay, and mortality were analyzed. Univariable and multivariable analyses were performed.

Results: There were 101,006 surgeries performed. The median age of patients was 0.5 years (interquartile range, 26 days to 3.2 years), and $1 \%$ of patients $(n=990)$ had heart block and placement of a pacemaker. Surgeries associated with the highest incidences of heart block and placement of a pacemaker included the double switch operation $(15.6 \%)$, tricuspid valve $(7.8 \%)$ and mitral valve $(7.4 \%)$ replacement, atrial switch with ventricular septal defect repair $(6.4 \%)$, and Rastelli operation $(4.8 \%)$. On multivariable analysis, after controlling for surgical complexity, other comorbidities, age at surgery, admission year, and clustering by institution, patients with heart block and placement of a pacemaker had higher odds of mortality (odds ratio, 1.67; 95\% confidence interval, 1.24-2.26; $P<.001$ ).

Conclusions: The incidence of postoperative heart block requiring permanent pacemaker placement immediately after congenital heart surgery is low $(1 \%)$. However, these patients have higher mortality even after adjusting for heart surgery complexity. (J Thorac Cardiovasc Surg 2016;152:197-202)

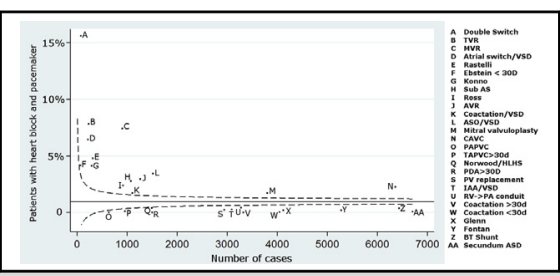

Funnel plot shows the incidence of heart block for each operation as a function of number of cases.

\section{Central Message}

We report the incidence of heart block requiring a pacemaker implant after surgery for congenital heart disease.

\section{Perspective}

The incidence of postoperative heart block requiring permanent pacemaker placement immediately after congenital heart surgery is low ( $1 \%$ on average). However, certain lesions are associated with significantly higher incidences, and the patients in whom heart block develops have significantly higher mortality and length of stay, even after adjusting for heart surgery complexity.

See Editorial Commentary page 203
Complete heart block has been reported as a complication of open surgery in patients with congenital heart disease since these procedures were first performed. ${ }^{1,2}$ Over the years, as surgical techniques improved, the incidence of postoperative chronic complete heart block decreased, and by the late 1960s, Fryda and colleagues ${ }^{3}$ reported an

\footnotetext{
From the ${ }^{\mathrm{a}}$ Division of Pediatric Cardiology, Department of Pediatrics, and ${ }^{\mathrm{b}}$ Division of Cardiothoracic Surgery, Department of Surgery, New York-Presbyterian/ Morgan Stanley Children's Hospital, Columbia University Medical Center, New York, NY.

Received for publication Oct 27, 2015; revisions received Feb 12, 2016; accepted for publication March 26, 2016; available ahead of print May 7, 2016.

Address for reprints: Leonardo Liberman, MD, New York-Presbyterian/Morgan Stan-

ley Children's Hospital, 3959 Broadway, CH-2 North, New York, NY 10032

(E-mail:11202@columbia.edu).

$0022-5223 / \$ 36.00$

Copyright (c) 2016 by The American Association for Thoracic Surgery

http://dx.doi.org/10.1016/j.jtcvs.2016.03.081
}

incidence of permanent postoperative heart block of $2 \%$. However, mortality for these patients was still high, with a range between $30 \%$ and $67 \% .^{1,3,4}$ To address this issue, surgeons started to place permanent pacemakers in all patients with postoperative complete heart block. ${ }^{5,6}$ Hofschire and colleagues ${ }^{6}$ reported a significant reduction in the episodes of Stokes-Adams attacks and sudden death in patients who underwent placement of a permanent pacemaker. In 1984, the first guidelines for permanent cardiac pacemaker implantation were published, ${ }^{7}$ and it was

Scanning this $\mathrm{QR}$ code will take you to the article title page.

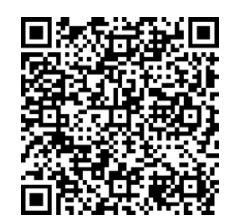




\section{Abbreviations and Acronyms \\ $\mathrm{CI}=$ confidence interval \\ ICD-9-CM = International Classification of \\ Diseases, 9th Revision, Clinical \\ Modification \\ IQR = interquartile range \\ OR $=$ odds ratio \\ RACHS-1 = Risk Adjusted Classification for \\ Congenital Heart Surgery, version 1 \\ VSD $=$ ventricular septal defect}

recommended that children with advanced second- or thirddegree atrioventricular block persisting 10 to 14 days after surgery receive a permanent pacemaker implant. In the subsequent revision of the guidelines, the recommended waiting period was shortened to 7 days. $^{8}$ Over the last 2 decades, reports from single or few institutions have been published describing the incidence of postoperative heart block. ${ }^{9-11}$ However, the true incidence of this complication in a large cohort of patients is unknown. We report a multicenter study of tertiary care institutions to determine the incidence of surgical heart block in the current era and to identify surgical risk factors for this complication.

\section{MATERIALS AND METHODS Study Design}

A retrospective cohort study was performed, using the Pediatric Health Information System database, to determine the incidence of complete heart block undergoing permanent pacemaker placement in pediatric patients after open surgery for congenital or acquired heart disease and to determine the effects of this outcome on mortality. This study was classified by the Columbia University Medical Center Institutional Review Board as nonhuman subjects research and was exempted from further review.

\section{Data Source}

Data for this study were obtained from the Pediatric Health Information System, an administrative database that contains inpatient observation data from 45 not-for-profit, tertiary care pediatric hospitals in the United States. These hospitals are affiliated with the Children's Hospital Association (Overland Park, Kan). Data quality and reliability are ensured through a joint effort between the Children's Hospital Association and participating hospitals. Participating hospitals provide discharge and encounter data, including demographics, diagnoses, and procedures. All data are deidentified at the time of data submission, and data are subjected to a number of reliability and validity checks before being included in the database.

\section{Study Population}

The database was queried for all children aged 18 years or less, discharged between January 1, 2004, and December 31, 2013, who underwent open surgery based on a Risk Adjusted Classification for Congenital Heart Surgery, version 1 (RACHS-1) score. ${ }^{12}$ Data collected included diagnosis of congenital heart diseased based on the International Classification of Diseases, 9th Revision, Clinical Modification (ICD-9-CM) codes, age at surgery, gender, and major comorbidities (genetic abnormalities and other chronic hematologic, renal, gastroenterologic, or neurologic conditions, as previously defined by Feudtner and colleagues ${ }^{13}$ ). Procedure codes were queried for all the surgical procedures based on the RACHS- 1 classification and all the surgical procedures for placement of a pacemaker and implantable defibrillator. Death during the hospitalization, length of stay, and costs per day were recorded. Patients with multiple surgical admissions were treated as if each admission was independent.

The information was analyzed for missing data. Missing data were defined as absence of age, cardiac diagnosis, or RACHS-1 procedure. Hospitals with less than 30 patients were excluded from analysis ( 3 centers, $\mathrm{n}=22$ patients).

\section{Predictors and Outcomes}

The primary outcome of interest was advanced second-degree (ICD-9CM: 426.12), third-degree (ICD-9-CM: 426.0), or unspecified heart block (ICD-9-CM: 426.10) in patients who underwent placement of a permanent ventricular or dual-chamber pacemaker or defibrillator. Patients who underwent a permanent pacemaker or defibrillator on the same date or before their RACHS-1 operation were excluded from further analysis.

The primary predictor of interest was the procedure done during the hospitalization that had the highest RACHS-1 score. Other variables considered included age at surgery, gender, RACHS-1 scoring, comorbidities (including genetic abnormalities and other chronic hematologic, renal, gastroenterologic, or neurologic conditions), year of operation, and admitting hospital.

The effects of the primary outcome on mortality, total hospital length of stay, and inpatient standardized costs per day were analyzed. Mortality was defined as in-hospital death. Standardized costs are adjusted for costs of living by hospital location, using the Centers for Medicare \& Medicaid Services Wage Index (http://www.cms.gov/Medicare/Medicare-Fee-forService-Payment/AcuteInpatientPPS/Wage-Index-Files.html) to allow comparison of resource use across institutions. All costs were further adjusted for inflation (to 2014 dollars), using the Medical Consumer Price Index (http://www.bls.gov/cpi/\#tables).

\section{Data Analysis}

Statistical analyses were conducted in Stata software, version 13 (StataCorp LP, College Station, Tex). Standard summary statistics were used, including means with standard deviations or medians with interquartile ranges (IQRs) for continuous variables, and frequencies with proportions for categoric variables. For each of 57 operation types (defined by RACHS- 1 coding), rates of heart block and pacemaker placement were calculated. These rates were compared with the overall incidence in the rest of the cohort by chi-square with Bonferroni correction. These rates were then graphically depicted for each procedure as a function of the number of cases in our cohort. Lines depicting the 5th and 95th binomial prediction limits were superimposed to create a funnel plot. Procedure types falling above or below these prediction limits were said to be associated with significantly high or low incidences, respectively.

To assess the association between predictor variables and mortality, we used chi-square, $t$ tests, and Wilcoxon rank-sum tests. To assess the association between predictor variables and length of stay and costs per day, we used Wilcoxon rank-sum tests and Spearman correlation. Variables with $P$ values of .10 or less in univariable analyses were evaluated together in multivariable analyses using logistic regression in the assessment of mortality and linear regression in the assessment of length of stay and costs per day. We clustered standard errors on institution to account for possible correlation between children treated within each center.

\section{RESULTS}

\section{Patient Characteristics}

A total of 102,271 hospitalizations (75,094 patients) from 42 hospitals were identified during the study period. Of 
these, $1265(1.2 \%)$ hospitalizations were excluded because the patients underwent placement of a permanent pacemaker $(\mathrm{n}=1161[92 \%])$ or defibrillator $(\mathrm{n}=104[8 \%])$ at the time or before the RACHS- 1 operation. These patients were older (3.6 years; IQR, $1.2-8.1$ years; $P<.001$ ) than patients receiving the device after the RACHS- 1 operation.

The remaining 101,006 hospitalizations (74,571 patients) were included for further analysis. The median age at the time of surgery was 0.5 years (IQR, 26 days to 3.2 years) with 61,405 patients $(61 \%)$ aged less than 1 year of age and 25,671 patients $(25 \%)$ aged less than 1 month. There were 55,835 male patients $(55 \%)$.

\section{Pacemaker Placement}

There were 1279 hospitalizations $(1.3 \%)$ in which patients underwent placement of a pacemaker or defibrillator during the hospitalization and after the RACHS-1 index operation. There were 4176 hospitalizations $(4.1 \%)$ in which patients were coded for advanced second- or thirddegree atrioventricular block. A total of 990 patients $(1 \%)$ had both heart block and a device placed, and they represent the main outcome variable analyzed. These patients underwent placement of a pacemaker $(n=947$, $96 \%)$ or an implantable defibrillator $(n=43,4 \%)$. The approach to the placement of the device was not well coded: There were $579(59 \%)$ epicardial devices, $173(17 \%)$ transvenous systems, and $238(24 \%)$ unknown route of pacing. The patients who had a transvenous system were older than the patients with an epicardial system (7.9 years, IQR, 3.4-13.5 years vs 5.3 months, IQR, 35 days to 1.6 years; $P<.001)$. The median time from the main RACHS-1 operation to the placement of the pacemaker was 9 days (IQR, 7-14 days).

Figure 1 shows the distributions of the procedures with the highest numbers of patients who underwent pacemaker placement for heart block. Figure 1 does not represent the risk of this outcome for each operation. Table 1 shows the incidence (and 95\% confidence intervals [CIs]) of being coded for heart block and having a permanent pacemaker placement in the operation types with the highest incidence of this complication. The surgeries associated with the highest risk of having heart block and permanent pacemaker placement were double switch operation $(15.6 \%)$, tricuspid valve $(7.8 \%)$ and mitral valve $(7.4 \%)$ replacement, atrial switch with ventricular septal defect (VSD) repair (6.4\%), Rastelli operation $(4.8 \%)$, neonatal Ebstein's repair (4.2\%), Konno operation (4.1\%), arterial switch operation with VSD repair (3.4\%), aortic valve replacement $(2.9 \%)$, subaortic resection $(2.8 \%)$, and Ross operation $(2.4 \%)$.

Tricuspid valvuloplasty and non-neonatal Ebstein's repair had a significantly high incidence of being coded for heart block $(5.9 \%$ and $10.8 \%$, respectively); however, patients with these lesions did not have a significantly high incidence of pacemaker placement ( $1.3 \%$ and $1.1 \%$, respectively).

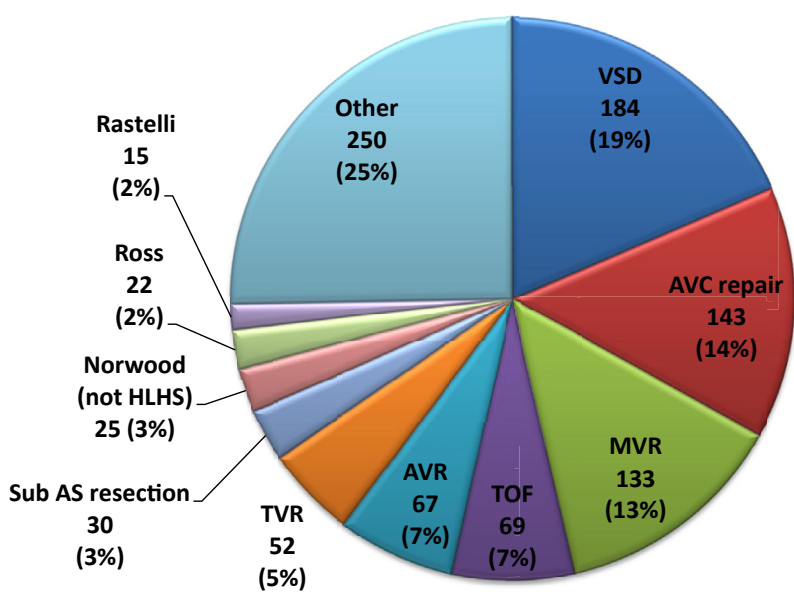

FIGURE 1. Distribution of the operations with placement of a permanent pacemaker for heart block after surgery $(\mathrm{n}=990)$. HLHS, Hypoplastic left heart syndrome; Sub AS, subaortic stenosis; TVR, tricuspid valve replacement; $A V R$, aortic valve replacement; TOF, tetralogy of Fallot; VSD, ventricular septal defect; $M V R$, mitral valve replacement; $A V C$, atrioventricular canal.

Other surgical procedures, including repair of secundum atrial septal defect, coarctation of the aorta, partial anomalous pulmonary venous return, patent ductus arteriosus repair, and Glenn and Fontan operations, had low incidences of heart block and a pacemaker placement rate that was statistically significantly lower than for the rest of the cohort $(P<.001)$. Figure 2 shows a funnel plot indicating the surgeries with high and low incidences of heart block with pacemaker placement, accounting for the number of patients undergoing each operation.

The patient's age, gender, and year of operation were not associated with postoperative heart block with pacemaker placement. Among the cardiac diagnoses, corrected transposition of the great arteries had the highest incidence of heart block with pacemaker placement $(4.1 \%, P<.001)$.

The median number of cases by center per year was 278 cases (IQR, 212-464), and the median number of cases per surgeon per year (after 2010) was 111 cases (IQR, 83-138). Center volume and surgeon volume were not associated with being coded for heart block and undergoing a pacemaker placement, even after controlling for RACHS-1 categories. The number of patients undergoing pacemaker implant for heart block after surgery varied between $0.77 \%$ and $1.1 \%$ each year without a particular trend $(P=.154)$.

\section{Mortality}

The overall mortality rate in the entire cohort was 3\% $(\mathrm{n}=3072)$. The presence of heart block and having a pacemaker implanted during the same hospitalization and after the RACHS-1 procedure were significantly associated with mortality. In univariable analyses (clustering standard errors by center), the presence of heart block and having a pacemaker implanted was associated with increased odds 
TABLE 1. Incidence of heart block with pacemaker placement by cardiac operation

\begin{tabular}{|c|c|c|c|c|c|}
\hline Surgery & Patients (n) & Pacemaker (n) & $\%$ & $\mathbf{9 5} \% \mathrm{CI}$ & $P$ value \\
\hline Double switch operation & 77 & 12 & 15.6 & $7.5-23.7$ & $<.001$ \\
\hline Tricuspid valve replacement & 230 & 18 & 7.8 & $4.3-11.3$ & $<.001$ \\
\hline Mitral valve replacement & 902 & 67 & 7.4 & $5.7-9.1$ & $<.001$ \\
\hline Atrial switch with VSD & 217 & 14 & 6.5 & $3.1-9.7$ & $<.001$ \\
\hline Rastelli operation & 313 & 15 & 4.8 & $2.4-7.1$ & $<.001$ \\
\hline Ebstein's repair $<30 \mathrm{~d}$ & 72 & 3 & 4.2 & $0-8.8$ & $<.001$ \\
\hline Konno operation & 290 & 12 & 4.1 & $1.8-6.4$ & $<.001$ \\
\hline ASO with VSD & 1517 & 52 & 3.4 & $2.5-4.3$ & $<.001$ \\
\hline Aortic valve replacement & 1262 & 37 & 2.9 & $2.0-3.9$ & $<.001$ \\
\hline Sub-AS resection & 1078 & 30 & 2.8 & $1.8-3.8$ & $<.001$ \\
\hline Ross operation & 918 & 22 & 2.4 & $1.4-3.4$ & $<.001$ \\
\hline CAVC repair & 6358 & 143 & 2.2 & $1.9-2.6$ & $<.001$ \\
\hline Mitral valvuloplasty & 3802 & 66 & 1.7 & $1.3-2.2$ & $<.001$ \\
\hline Coarctation with VSD & 1107 & 19 & 1.7 & $1.0-2.5$ & .01 \\
\hline
\end{tabular}

$P$ values compare the incidence of heart block for a specific procedure with that observed in the rest of the study cohort. $C I$, Confidence interval; VSD, ventricular septal defect; $A S O$, arterial switch operation; Sub-AS, subaortic stenosis; $C A V C$, complete atrioventricular canal.

of mortality (odds ratio [OR], 2.0; 95\% CI, 1.51-2.74; $P<.001)$. In multivariable analysis, the presence of heart block and having a pacemaker implanted was still associated with increased mortality (OR, 1.67; 95\% CI, 1.242.26; $P<.001$ ), after controlling for RACHS-1 category, other comorbidities (genetic abnormalities and other chronic hematologic, renal, gastroenterologic, or neurologic conditions), age at surgery, and admission year.

\section{Resource Use}

The median length of stay for patients who had heart block and underwent placement of a pacemaker was longer than for the rest of the cohort (20 days, IQR, 12-41 vs 8 days, IQR, 4-17) $(P<.001)$. In multivariable analyses, the presence of heart block and a pacemaker was associated with 2.1 times increase in length of stay (95\% CI, 2.0-2.3; $P<.001$ ), after controlling for the RACHS-1 category, age at surgery, and presence of other comorbidities (genetic abnormalities and other chronic hematologic, renal, gastroenterologic, or neurologic conditions) clustering by institution, and after censoring for death.

The median inpatient total hospital cost for patients who were coded for heart block and underwent placement of a pacemaker was $\$ 120,377$ (IQR, \$78,900-\$212,281), in 2014 dollars. The median hospital cost for the rest of the cohort was \$47,841 (IQR, \$30,597-\$93,457) $(P<.001)$. The median hospital cost per day was $\$ 5907$ (IQR, \$4532-\$7793), which was not statistically different in patients with or without heart block and placement of a pacemaker on univariable or multivariable analysis.
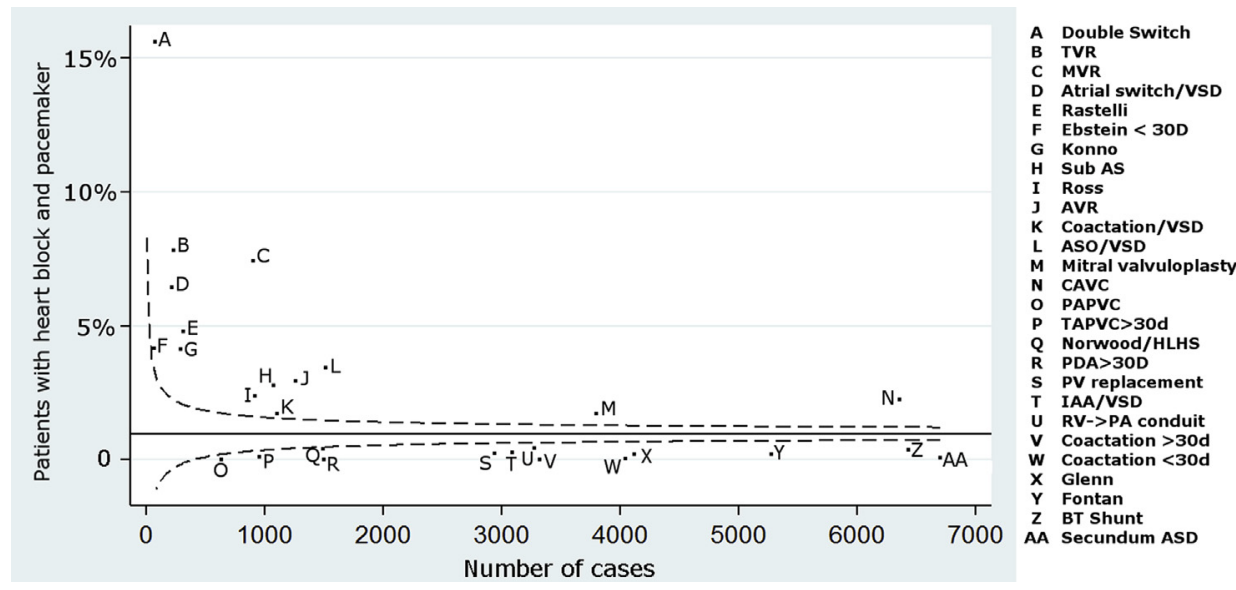

FIGURE 2. This funnel plot displays the incidence of heart block with pacemaker placement for each of 57 operations as a function of the number of cases in the cohort. The horizontal solid line represents the average incidence across all operation subtypes. The dashed lines represent the 5th and 95 th binomial prediction limits. Procedure types falling above or below these limits were said to be associated with significantly high or low incidences, respectively. TVR, Tricuspid valve replacement; $M V R$, mitral valve replacement; $V S D$, ventricular septal defect; Sub $A S$, subaortic stenosis; $A V R$, aortic valve replacement; $A S O$, arterial switch operation; $C A V C$, complete atrioventricular cannal; $P A P V C$, partial anomalous pulmonary venous connection; TAPVC, total anomalous pulmonary venous connection; $H L H S$, hypoplastic left heart syndrome; $P D A$, patent ductus arteriosus; $R V$, right ventricle; $P A$, pulmonary artery; $I A A$, interrupted aortic arch; $P V$, pulmonary valve; $B T$, Blalock-Tausig shunt; $A S D$, atrial septal defect. 


\section{DISCUSSION}

In this multicenter study, we analyzed data from more than 100,000 operations at 42 children's hospitals over a 10 -year period to provide insights into the incidence and impacts of heart block requiring permanent pacemakers for children undergoing open surgery in the current era. Previous reports have been limited to the experiences of individual centers or select lesions. ${ }^{9-11}$ In this study, we not only examined the majority of congenital heart operations but also accounted for intercenter variation and trends over time.

We were able to determine the incidence of this complication overall $(1 \%)$ and to explore the risks associated with each specific procedure. We found that the risk of this complication was highest for children undergoing the double switch operation (15\%), followed by tricuspid and mitral valve replacements ( $\sim 7 \%$ for each). Contrary to a common belief, isolated VSD and tetralogy of Fallot surgeries did not pose a particularly high risk for developing this complication when compared with other cardiac lesions, although the absolute number of patients undergoing these operations who require pacemaker placement is high (Figure 1) because of the frequency of these operations. As expected, cardiac surgeries not involving the crux of the heart, such as coarctation of the aorta repair, Glenn or Fontan operations, pulmonary valve replacement, and secundum atrial septal defect, among others, had low incidences of heart block and pacemaker placement.

Prior reports have shown low or no incidence of postoperative heart block after repair of Ebstein's disease ${ }^{14,15}$ or tricuspid valvuloplasty. ${ }^{16,17}$ Of note, in our cohort, patients undergoing non-neonatal Ebstein's repair and tricuspid valvuloplasty had high incidences of heart block. However, these patients did not have a significantly high incidence of permanent pacemaker placement. This might suggest that there is a higher rate of conduction recovery in these patients. One might speculate that perhaps these patients should be observed for longer periods of time before placement of permanent pacemakers.

It has been well described that hospital mortality after repair of congenital heart disease increases with increased surgical complexity. ${ }^{18}$ Our study validated this finding (data not shown). Furthermore, we demonstrate that patient hospital mortality was associated with having heart block and undergoing placement of a permanent pacemaker, even after controlling for RACHS-1 category and other chronic comorbidities.

Prior studies have shown that hospital surgical volume is associated with hospital mortality, ${ }^{19-22}$ and this metric has gained increasing attention over recent years as a potential measure of experience. Our data confirmed the inverse relationship between center volume and mortality; the risk-adjusted OR for mortality was 0.77 (95\% CI,
0.72-0.83) for hospitals performing more than 300 operations per year. However, the development of heart block requiring a pacemaker implant was not associated with hospital volume. Likewise, this postoperative complication was not associated with surgeon annual volume, indicating that center or surgeon experience might not be an important determinant of the development of this complication, which is probably inevitable in certain patients.

\section{Study Limitations}

This study has limitations inherent to its retrospective nature and the fact that it is based on administrative data. In addition, the conclusions rely on several assumptions. First, we assumed that patients who were coded for second- or third-degree atrioventricular block and who underwent pacemaker placement during their hospitalization, but after the reference operation, developed this complication as a result of the antecedent surgery. It is possible that some of these patients had preoperative heart block. However, one would assume that most patients with preoperative heart block should have had pacemaker placement on the same day as their reference surgery and would have been excluded from the analysis. Second, we only consider patients who underwent postoperative pacemaker placement before discharge from the hospital. There might be patients who develop late changes in conduction that result in pacemaker placement after hospital discharge. Although, in our clinical experience, ${ }^{23}$ these patients are few, this might increase the incidences estimated in the current study. Finally, these data include only those that are captured by administrative coding; therefore, clinical information such as electrocardiogram characteristics, ventricular function, hemodynamic parameters, and so forth are not available. Follow-up studies using clinical registries with more granularity would add increased depth.

\section{CONCLUSIONS}

In this multicenter study, examining records for more than 100,000 children from 42 institutions who underwent open surgery, we found that $1 \%$ of the patients had heart block and underwent placement of a permanent pacemaker. This complication increases hospital mortality.

\section{Conflict of Interest Statement}

Authors have nothing to disclose with regard to commercial support.

\section{References}

1. Lillehei CW, Sellers RD, Bonnabeau RC, Eliot RS. Chronic postsurgical com plete heart block. J Thorac Cardiovasc Surg. 1963;46:436.

2. McGoon DC, Ongley PA, Kirklin JW. Surgical heart block. Am J Med. 1964;37: 749.

3. Fryda RJ, Kaplan S, Helmsworth JA. Postoperative complete heart block in children. Br Heart J. 1971;33:456. 
4. Hurwitz RA, Riemenschneider TA, Moss AJ. Chronic postoperative heart block in children. Am J Cardiol. 1968;21:185.

5. Harris PD, Bowman FO Jr, Griffiths SP. Implantation of a synchronous pacing unit in a seven-month-old infant. J Thorac Cardiovasc Surg. 1966;52:277.

6. Hofschire PJ, Nicoloff DM, Moller JH. Postoperative complete heart block in 64 children treated with and without cardiac pacing. Am J Cardiol. 1977;39:559.

7. Frye RL, Collins JJ, DeSanctis RW, Dodge HT, Dreifus LS, Fisch C, et al. Guidelines for permanent cardiac pacemaker implantation. J Am Coll Cardiol. 1984;4: 434-42.

8. Gregoratos G, Cheitlin MD, Conill A, Epstein AE, Fellows C, Ferguson TB, et al. ACC/AHA Guidelines for Implantation of Cardiac Pacemakers and Antiarrhythmia Devices: Executive Summary. Circulation. 1998;97:1325-35.

9. Weindling SN, Saul JP, Gamble WJ, Mayer JE, Wessel D, Walsh EP. Duration of complete atrioventricular block after congenital heart disease surgery. Am J Cardiol. 1998;82:525-7.

10. Goldman BS, Williams WG, Hill T, Hesslein PS, McLaughlin PR, Trusler GA, et al. Permanent cardiac pacing after open heart surgery: congenital heart disease. Pacing Clin Electrophysiol. 1985;8:732-9.

11. Anderson JB, Czosek RJ, Knilans TK, Meganathan K, Heaton P. Postoperative heart block in children with common forms of congenital heart disease: results from the KID database. J Cardiovasc Electrophysiol. 2012;23:1349-54.

12. Jenkins KJ, Gauvreau K, Newburger JW, Spray TL, Moller JH, Iezzoni LI. Consensus-based method for risk adjustment for surgery for congenital heart disease. J Thorac Cardiovasc Surg. 2002;123:110-8.

13. Feudtner C, Hays RM, Haynes G, Geyer JR, Neff JM, Koepsell TD. Deaths attributed to pediatric complex chronic conditions: national trends and implications for supportive care services. Pediatrics. 2001;107:E99.

14. Ullmann MV, Born S, Sebening C, Gorenflo M, Hulmer HE, Hagl S. Ventricularization of the atrialized chamber: a concept of Ebstein's anomaly repair. Ann Thorac Surg. 2004;78:918-25.

15. Da Silva JP, Baumgratz JF, Da Fonseca L, Franchi SM, Lopes LM, Tavares GM, et al. The cone reconstruction of the tricuspid valve in Ebstein's anomaly. The operation: early and midterm results. J Thorac Cardiovasc Surg. 2007;133: 215-23.

16. McCarthy PM, Bhudia SK, Rajeswaran J, Hoercher HJ, Lytle BW, Cosgrove DM, et al. Tricuspid valve repair: durability and risk factors for failure. J Thorac Cardiovasc Surg. 2004;127:674-85.

17. Singh SK, Tang GH, Maganti MD, Armstrong S, Williams WG, David TE, et al. Midterm outcomes of tricuspid valve repair versus replacement for organic tricuspid disease. Ann Thorac Surg. 2006;82:1735-41.

18. Jenkins KJ. Risk adjustment for congenital heart surgery: the RACHS-1 method. Semin Thorac Cardiovasc Surg Pediatr Card Surg Annu. 2004;7: $180-4$.

19. Vinocur JM, Menk SJ, Connett J, Moller JH, Kochilas LK. Surgical volume and center effects on early mortality after pediatric cardiac surgery: 25-year North American experience from a multi-institutional registry. Pediatr Cardiol. 2013; 34:1226-36.

20. Jenkins KJ, Newburger JW, Lock JE, Davis RB, Coffman GA, Iezzoni LI. In-hospital mortality for surgical repair of congenital heart defects: preliminary observations of variation by hospital caseload. Pediatrics. 1995;95: 323-30.

21. Pasquali SK, Li JS, Burstein DS, Shenq S, O’Brien SM, Jacobs ML, et al. Association of center volume with mortality and complications in pediatric heart surgery. Pediatrics. 2012;129:e370-6.

22. Anderson BR, Ciarleglio AJ, Cohen, Lai WW, Neidell M, Hall M, et al. The Norwood operation: relative effects of surgeon and institutional volumes on outcomes and resource utilization. Cardiol Young. 2015;14:1-10.

23. Liberman L, Pass RH, Hordof AJ, Spotnitz HM. Late onset of heart block after open heart surgery for congenital heart disease. Pediatr Cardiol. 2008;29: 56-9.

Key Words: congenital heart disease, heart block, pacemaker 